\title{
Male-killing Wolbachia do not protect Drosophila bifasciata against viral infection
}

Ben Longdon ${ }^{1 *}$, Daniel K Fabian ${ }^{3,4}$, Gregory DD Hurst ${ }^{2}$, Francis M Jiggins ${ }^{3}$

\begin{abstract}
Background: Insect symbionts employ multiple strategies to enhance their spread through populations, and some play a dual role as both a mutualist and a reproductive manipulator. It has recently been found that this is the case for some strains of Wolbachia, which both cause cytoplasmic incompatibility and protect their hosts against viruses. Here, we carry out the first test as to whether a male-killing strain of Wolbachia also provides a direct benefit to its host by providing antiviral protection to its host Drosophila bifasciata. We infected flies with two positive sense RNA viruses known to replicate in a range of Drosophila species (Drosophila C virus and Flock House virus) and measure the rate of death in Wolbachia positive and negative host lines with the same genetic background.

Results: Both viruses caused considerable mortality to D. bifasciata flies, with Drosophila C virus killing $43 \%$ more flies than the uninfected controls and Flock House virus killing $78 \%$ more flies than the uninfected controls. However, viral induced mortality was unaffected by the presence of Wolbachia.

Conclusion: In the first male-killing Wolbachia strain tested for antiviral effects, we found no evidence that it conferred protection against two RNA viruses. We show that although antiviral resistance is widespread across the Wolbachia phylogeny, the trait seems to have been lost or gained along some lineages. We discuss the potential mechanisms of this, and can seemingly discount protection against these viruses as a reason why this symbiont has spread through Drosophila populations.
\end{abstract}

\section{Background}

Maternally transmitted bacterial symbionts are extremely common in insects, with over half of all species estimated to be infected by bacteria from the genus Wolbachia alone [1]. Because maternal inheritance is often imperfect, and there is commonly a direct physiological cost to infection associated with presence of the bacteria, these infections can only be maintained where they increase either the survival or production of female hosts [2]. Some symbionts become parasites that manipulate the reproduction of their hosts to enhance their own transmission [3]. For example, many distort the sex ratio of the host towards females - the transmitting sex - to aid their spread [4-6]. Others act as mutualists, increasing the survival or reproductive success of their

\footnotetext{
* Correspondence: B.Longdon@ed.ac.uk

${ }^{1}$ Institute of Evolutionary Biology, and Centre for Immunity, Infection and Evolution, University of Edinburgh, Ashworth Labs, Kings Buildings, West Mains Road, Edinburgh, EH9 3JT, UK

Full list of author information is available at the end of the article
}

hosts, and therefore the number of offspring to which they are transmitted [7]. Some mutualists are essential for the host to survive and reproduce (primary symbionts) [8], while others play non-essential facultative roles and typically only infect a subset of the population (secondary symbionts $[7,9]$ ).

A number of recent studies have found secondary symbionts providing the host with protection against parasites and pathogens [10]. In aphids various bacterial symbionts confer protection to parasitoid wasps [11-13] and fungi [14], while Spiroplasma bacteria provide protection from nematodes in Drosophila neotestacea [15] and parasitoids in Drosophila hydei [16]. Recently, Wolbachia has been shown to make species of Drosophila and mosquitoes resistant to RNA viruses [17-22]. It can also make $D$. melanogaster more tolerant to viral infection, as the survival of flies infected with flock house virus (FHV) increased despite there being no effect on viral titres [18]. This protection against viruses is effective against a remarkably diverse range of single-

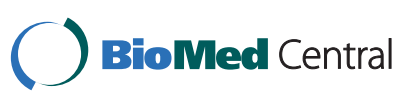


stranded positive-sense RNA viruses, including; Dicistroviridae (Drosophila $C$ virus and Cricket paralysis virus), Nodaviridae (Flock House virus), Picorna-like viruses (Nora virus), Togaviridae (Chikungunya virus) and Flaviviridae (Dengue virus and West Nile virus) $[17,18,20,22,23]$.

Symbionts can sometimes employ multiple strategies to enhance their spread through populations. Rickettsia in whiteflies act both to directly increase host fitness and distort the sex ratio towards the production of female offspring [24]. It has recently been shown that the same strain of Wolbachia can both act as both a mutualist and a reproductive manipulator; in Drosophila simulans, strains of Wolbachia that induce strong cytoplasmic incompatibility also protect the host from viral infection [19]. Such dual strategies have the potential to explain several puzzling aspects of symbiont biology. For example, symbionts that cause cytoplasmic incompatibility are extremely common, despite them only being able to invade populations when they exceed a threshold prevalence $[2,25,26]$. This restrictive condition for invasion can disappear if the bacterium is also a mutualist [2]. If symbionts are maintained in populations by cytoplasmic incompatibility, theory predicts that there are no stable equilibria below $50 \%$, and yet observed prevalence for Wolbachia in D. melanogaster are commonly below $50 \%$ $[27,28]$. This has led to the prediction that such symbionts must also carry some unknown benefit to host fitness [29], and recent models have suggested natural enemy resistance can both eliminate any threshold for invasion and stabilize low prevalence Wolbachia infections [30]. Similarly, male-killing bacteria spread when the death of a male benefits its sisters who will transmit the infection, and this will only occur when there are antagonistic sibling interactions such as cannibalism or competition [4]. However, some male-killers have been reported from species where eggs are laid singly [31], so sibling interactions are of low intensity. Again, this could be explained if these bacteria have other effects, such as increasing host resistance to pathogens. The high prevalence of symbionts within and across species [32] could therefore be result of such symbionts that 'employ' multiple strategies, and may help explain their apparent success in invading new host populations or host species.

In this study we have tested whether D. bifasciata infected with a male-killing strain of Wolbachia have greater protection from viral pathogens. This strain of Wolbachia naturally infects 5-7\% of female D. bifasciata resulting in close to $100 \%$ female broods at $18^{\circ} \mathrm{C}$ [33]. At elevated temperatures, infected males can be produced, and then the bacteria cause weak cytoplasmic incompatibility when crossed to uninfected females [33]. In this study we examine whether this bacterium has a third phenotype by testing whether it confers protection from two RNA viruses.

The first virus we used was Drosophila C virus (DCV), a positive sense RNA virus in the family Dicistroviridae [34] that naturally infects $D$. melanogaster in the wild $[35,36]$. DCV commonly infects laboratory stocks of other Drosophila species [37], and can replicate when injected into a wide range of insects [38]. Secondly we used Flock House virus (FHV), a positive sense RNA virus in the family Nodaviridae [39]. It is not a natural pathogen of Drosophila (having been isolates from a coleopteran [40]), but will replicate in a broad range of insects and other taxa [41-44]. Wolbachia has been reported to increase the survival of $D$. melanogaster infected with both of these viruses $[17,18]$.

\section{Methods}

The Wolbachia-infected line of Drosophila bifasciata was collected in Japan in 1998 [33]. Since then (>140 generations) they have since been maintained by backcrossing infected females to males from an isofemale uninfected line present in the lab for 20 years. The two lines therefore have the same nuclear genetic background. Because infected flies were maintained using male flies from the uninfected stock, other aspects of the flies (such as any commensal flora) will also be similar. The Wolbachia infection rate was 100\% (no males were observed in the infected line). The flies were reared on agar-malt medium at $\sim 18^{\circ} \mathrm{C}$.

We used reverse transcription (rt) PCR to check that the fly stocks we were using were not infected with DCV or FHV before the experiment. Total RNA was extracted from 40 individuals per line using Trizol reagent (Invitrogen Corp, San Diego, CA, USA) as described previously [45]. RNA was then reverse-transcribed with Promega Goscript reverse transcriptase (Promega Corp, Madison, WI, USA) using random hexamer primers. PCR was carried out on each line using DCV (kindly provided by Darren Obbard) and FHV primers [46] (DCV1290F 5'- GATGGTGTTGGCTCTGAACAGATG-3', DCV1590R 5'-CAACTGTATCT TCCAATGCACCCTG-3' FHV RNA 1\&3 F 5'-GGACCGAAGTGCGGTGATG-3', FHV RNA 1\&3 R 5'CAGTTTTGCGGGTGGGGGG-3') with a touchdown PCR cycle and the viral isolates used for injections as positive controls. The FHV primer pair are located in conserved regions (based on alignment to the related Black Beetle virus and Boolara virus) as are the DCV primers (based on an alignment to another DCV isolate: Darren Obbard personal communication) so should amplify any similar viruses if present.

We then tested the effect of fly Wolbachia infection status on viral pathogenicity. The viral isolates have been described previously $[36,46]$ (kindly provided by 
Luis Texiera) and were prepared as in [18]. We injected virgin females aged between 4 and 10 days old with $69 \mathrm{nl}$ of virus into the abdomen of the fly using a Nanoject II (Drummond scientific, Bromall, PA, USA). The viruses were injected at a tissue culture infective dosage $_{50}$ of $1.35 \times 10^{6} \mathrm{TCID}_{50}$ in $69 \mathrm{nl}$ for FHV and 1000 TCID $_{50}$ in $69 \mathrm{nl}$ for DCV.

To produce the virus, Schneider Drosophila line 2 (DL2) cells were cultured at $26.5^{\circ} \mathrm{C}$ in Schneider's Drosophila Medium (Invitrogen) supplemented with $10 \%$ Fetal Bovine Serum, 2mM L-Glutamine, $100 \mathrm{U} / \mathrm{ml}$ penicillin, and $100 \mu \mathrm{g} / \mathrm{ml}$ streptomycin (all Invitrogen). The cells were infected with DCV, and after they showed cytopathic effect they were filtered through a $0.45 \mu \mathrm{m}$ filter and centrifuged at $13.500 \mathrm{rpm}$ for 10 minutes to remove any bacteria or cellular components. Aliquots of a $10^{-4}$ dilution of the virus suspension were prepared using $50 \mathrm{mM}$ TE buffer and frozen at $-80^{\circ} \mathrm{C}$. To calculate the infectivity of the virus, the Tissue Culture Infective Dose $50\left(\mathrm{TCID}_{50}\right)$ was calculated. Starting from the $10^{-4}$ dilution, serial dilutions to $10^{-10}$ were made in Schneider's medium, and each dilution was added to 8 wells of a plate. After 7 days the wells were examined and classed as "infected" when cell death and cytopathic effects were clearly visible. The $\mathrm{TCID}_{50}$ was calculated by the Reed-Muench end-point method [47]. The Poisson distribution was used to get the number of infective units per $\mathrm{ml}(\mathrm{IU} / \mathrm{ml})$ [48]. The experiment was done twice to ensure the estimates of the $\mathrm{TCID}_{50}$ were consistent.

As a negative control we also injected flies with Drosophila Ringer's solution [49] for the DCV experiment and Drosophila Ringer's solution diluted 1:2 with Tris $50 \mathrm{mM} \mathrm{pH} 7.5$ for the FHV experiment. The different negative controls reflect how the viral isolate was diluted. After injection, flies were kept in vials of agarsugar medium at $\sim 18^{\circ} \mathrm{C}$.

The flies were examined each day and the number of dead individuals in each vial was recorded. The effect of Wolbachia on survival rates was analysed using a Cox's proportional hazards mixed effect model, which accounted for between vial variation in survival rates. The hazard for the $i$ th individual from vial $j$ at time $t$ was modelled as:

$$
H_{i j}(t)=H_{0}(t) e^{X_{i} \beta+b_{j}}
$$

Where $H_{0}(t)$ is the baseline hazard at time $t, X_{i}$ is a vector of the fixed effects, $\beta$ is the corresponding vector of coefficients, and $b_{j}$ is a random effect of vial $j$. The fixed effects consisted of treatment (virus or negative), Wolbachia (infected or uninfected) and their interaction. Flies alive at the end of the experiment were censored.
The model was fitted by maximum likelihood using the coxme package in $\mathrm{R}$ ( $\mathrm{R}$ Foundation for Statistical Computing, Vienna, Austria).

\section{Results and discussion}

DCV: Having established that neither of the D. bifasciata lines tested positive for DCV-like viruses by rtPCR, 454 flies were injected with DCV (Additional file 1 ), and their mortality recorded over 16 days (Figure $1 \mathrm{a})$. DCV caused considerable mortality $(z=-4.32$, $P<0.001$ ), with the death rate of infected flies accelerating after ten days, such that $59 \%$ of the DCV injected flies had died by day 16 in comparison to $16 \%$ in the uninfected controls. However, the presence of Wolbachia did not affect the rate at which DCV kills flies (Wolbachia $\mathrm{x}$ treatment interaction: $z=0.23, P=0.82$ ), nor was there an overall effect of Wolbachia on survival $(z=0.51, P=0.61)$.

FHV: The results from the FHV experiment were similar. In this experiment 539 flies were injected (Additional file 1), and their mortality recorded over 12 days (Figure $1 \mathrm{~b}$ ). At the end of this time period $88 \%$ of the FHV infected flies were dead compared to $10 \%$ of the uninfected controls $(z=-8.72, P<0.001)$. Again the presence of Wolbachia had no affect on the rate at which FHV killed flies (Wolbachia $\mathrm{x}$ treatment interaction: $z=0.95, P=0.34$ ), nor was there any main effect of $\mathrm{Wol}$ bachia $(z=-0.29, P=0.77)$. Neither of the fly lines tested positive for FHV-like viruses by rtPCR.

It has recently become clear that secondary symbionts have often evolved multiple strategies to spread through host populations, and tests on a small number of $\mathrm{Wol}$ bachia strains have suggested that they may commonly play a dual role as a mutualist and reproductive parasite [19]. For the first time we have tested a male-killing strain of Wolbachia for antiviral effects, and we found it does not protect its host from the two RNA viruses we used. The number of other Wolbachia strains that have been examined for antiviral effects is still small, but the majority of these have provided protection against viruses. For example, in Drosophila, of the five Wolbachia strains tested, three have antiviral effects $(w \mathrm{Mel}$ and the mutant $w$ MelPop from D. melanogaster, and $w \mathrm{Au}$ and $w \mathrm{Ri}$ from $D$. simulans) [17-19]. Our results suggest that Wolbachia strains that do not protect their hosts against viruses may be common, and that each strain will require independent evaluation.

There are a number of possible explanations as to why many Wolbachia strains provide antiviral protection, whereas the $D$. bifasciata male killer strain does not. The difference could be caused by genes in the host, but results from other species suggest that this may not be the most likely explanation, as $w \mathrm{Mel}$ retained its 
a

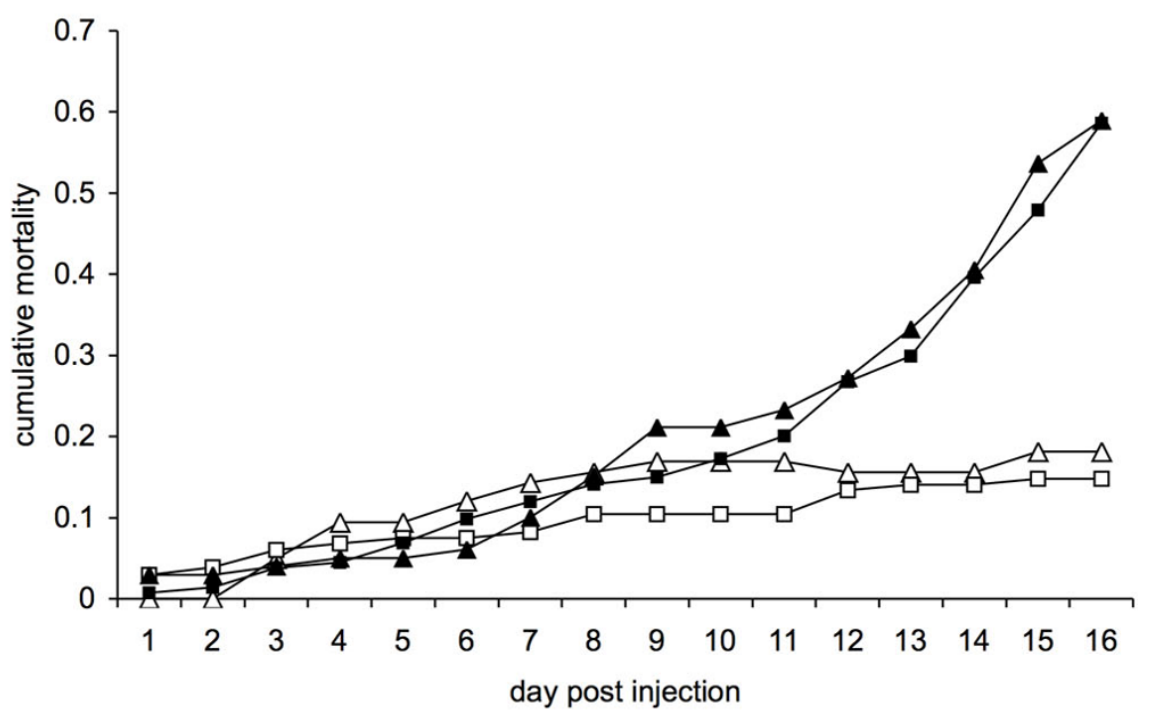

FHV

b

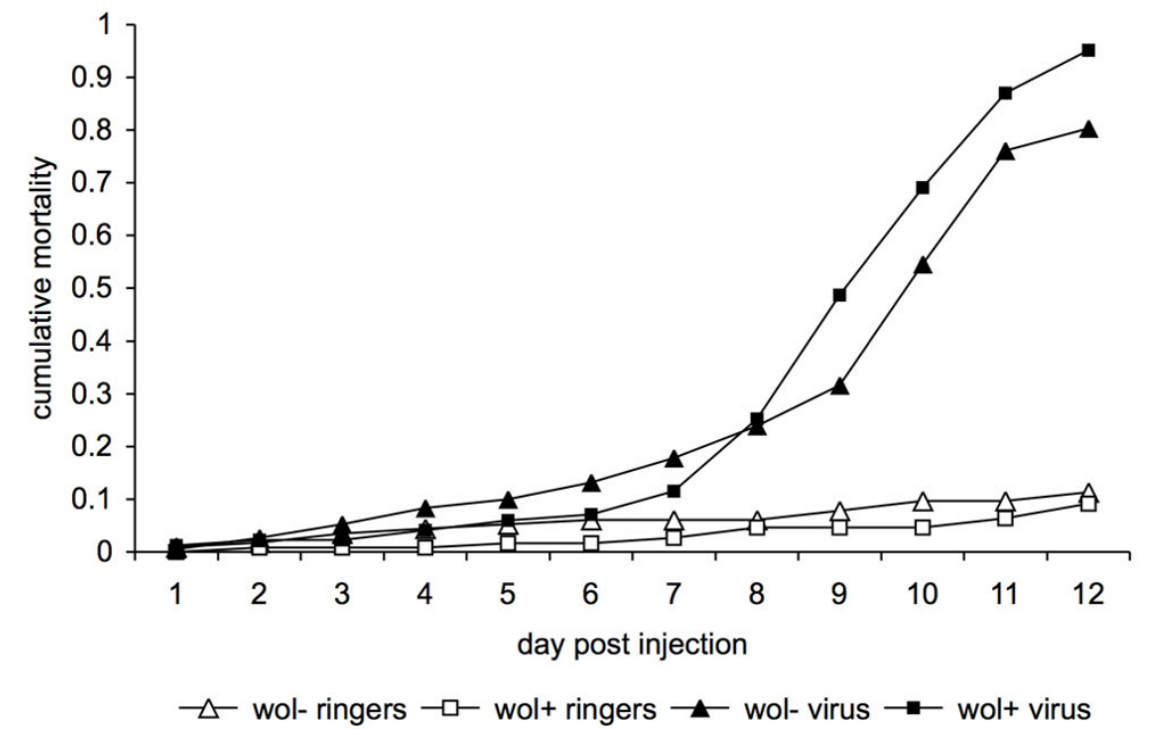

Figure 1 Cumulative mortality following injection with DCV (a) or FHV (b). Flies were Wolbachia infected (squares) or uninfected (triangles). Filled points represent viral injected and unfilled points control injected flies.

antiviral effect even when it was transferred between different dipteran families [20]. We may also have picked two viruses not affected by this strain of bacterium, but again results from other Wolbachia strains suggest that protection is effective against a diverse range of RNA viruses with positive sense genomes $[17,18,20,23]$. Therefore, perhaps the most likely reason that the $D$. bifasciata male killer may lack the antiviral effect seen in other strains is due to genetic factors in the bacteria.
Phylogenies of Wolbachia place the D. bifasciata male killer within the A clade, along with the other Wolbachia strains in Drosophila that offer protection against viruses [33,50,51]. In contrast, the Wolbachia strains from mosquitoes with antiviral effects belong to the $B$ clade $[21,23]$. The lack of association between this trait and the bacterial phylogeny suggests that the trait has been lost or gained on some lineages. This is unsurprising as the Wolbachia genome is known to recombine 
[52,53] and contains mobile phage [54]. In Hamiltonella defensa, the only case where the genetic basis of symbiont-mediated protection is known, a protection of aphids from parasitoid wasps is encoded on genes carried by a phage [55].

Regardless of whether host or bacterial genes determine whether different strains have antiviral effects, it is possible that these genes may not encode the antiviral factors themselves, but may simply control bacterial density. In both D. simulans [19] and Aedes albopictus [22] the Wolbachia strains offering the greatest protection to viruses have significantly greater densities of Wolbachia than those that did not.

In many cases the spread of male-killing bacteria through host populations is surprising. Male-killing bacteria are only expected to invade insect populations when the death of males benefits the surviving females who will transmit the infection to their offspring [4]. For example, the females may gain resources by eating their dead brothers or avoiding competing with them for resources. In species like ladybird beetles, the eggs are laid in clutches and there are strong antagonistic interactions between siblings. In other species, like Drosophila and some butterflies [31], the benefits of killing males are less obvious and it is possible that the bacteria may employ other strategies to aid their spread. However, we have found that in the case of D. bifasciata it seems the spread of the male-killer has not been aided by any antiviral effect against the two viruses examined here.

\section{Author contributions}

BL, GDDH and FMJ designed the study. BL and DKF carried out the experimental work. BL and FMJ analysed the data and drafted the manuscript with comments from GDDH and DKF. All authors read and approved the final manuscript.

\section{Additional material}

Additional file 1: Number of flies injected per treatment, figure in brackets is number of vials per treatment. There was a mean of 19 flies per vial.

\begin{abstract}
Acknowledgments
We thank Luis Texieria and Darren Obbard for providing viral stocks and primers. This work was supported by a BBSRC PhD studentship awarded to BL and a Royal Society Fellowship and Wellcome Trust grant (WT081279MA) to FMJ. We thank two anonymous reviewers for helpful comments. This article has been published as part of BMC Microbiology Volume 11 Supplement 1, 2012: Arthropod symbioses: from fundamental studies to pest and disease mangement. The full contents of the supplement are available online at http://www.biomedcentral.com/1471-2180/12?issue=S1.
\end{abstract}

\section{Author details}

${ }^{1}$ Institute of Evolutionary Biology, and Centre for Immunity, Infection and Evolution, University of Edinburgh, Ashworth Labs, Kings Buildings, West
Mains Road, Edinburgh, EH9 3JT, UK. ${ }^{2}$ Institute of Integrative Biology, University of Liverpool, Liverpool, L69 7ZB, UK. ${ }^{3}$ Department of Genetics, University of Cambridge, Cambridge, CB2 3EH, UK. ${ }^{4}$ Current address: Institute of Population Genetics, Vetmeduni Vienna, Veterinaerplatz 1, A-1210 Vienna, Austria.

\section{Competing interests}

The authors declare they have no competing interests.

Published: 18 January 2012

\section{References}

1. Hilgenboecker $K$, Hammerstein $P$, Schlattmann $P$, Telschow A, Werren JH: How many species are infected with Wolbachia?-A statistical analysis of current data. FEMS Microbiol Lett 2008, 281:215-220.

2. Fine PEM: Dynamics of symbiote-dependent cytoplasmic incompatibility in culicine mosquitos. Journal of Invertebrate Pathology 1978, 31:10-18.

3. Engelstadter J, Hurst GDD: The ecology and evolution of microbes that manipulate host reproduction. Annual Review of Ecology Evolution and Systematics 2009, 40:127-149.

4. Hurst GDD, Jiggins FM: Male-killing bacteria in insects: mechanisms, incidence, and implications. Emerging Infectious Diseases 2000, 6:329-336.

5. Rousset F, Bouchon D, Pintureau B, Juchault P, Solignac M: Wolbachia endosymbionts responsible for various alterations of sexuality in arthropods. Proceedings of the Royal Society of London Series B-Biological Sciences 1992, 250:91-98.

6. Stouthamer R, Luck RF, Hamilton WD: Antibiotics cause parthenogenetic trichogramma (Hymenoptera, Trichogrammatidae) to revert to sex. Proceedings of the National Academy of Sciences of the United States of America 1990, 87:2424-2427.

7. Buchner P: Endosymbiosis of Animals with Plant Microorganisms. New York: Interscience, Inc; 1965.

8. Douglas AE: Nutritional interactions in insect-microbial symbioses: aphids and their symbiotic bacteria buchnera. Annual Review of Entomology 1998, 43:17-37.

9. Tsuchida T, Koga R, Shibao H, Matsumoto T, Fukatsu T: Diversity and geographic distribution of secondary endosymbiotic bacteria in natural populations of the pea aphid, Acyrthosiphon pisum. Molecular Ecology 2002, 11:2123-2135.

10. Hurst GDD, Hutchence KJ: Host defence: getting by with a little help from our friends. Current Biology 2010, 20:R806-R808.

11. Oliver KM, Moran NA, Hunter MS: Variation in resistance to parasitism in aphids is due to symbionts not host genotype. Proceedings of the National Academy of Sciences of the United States of America 2005, 102:12795-12800.

12. Oliver KM, Russell JA, Moran NA, Hunter MS: Facultative bacterial symbionts in aphids confer resistance to parasitic wasps. Proceedings of the National Academy of Sciences of the United States of America 2003, 100:1803-1807.

13. Vorburger C, Gehrer L, Rodriguez P: A strain of the bacterial symbiont Regiella insecticola protects aphids against parasitoids. Biology Letters 2010, 6:109-111.

14. Scarborough $\mathrm{CL}$, Ferrari J, Godfray $\mathrm{HCJ}$ : Aphid protected from pathogen by endosymbiont. Science 2005, 310:1781-1781.

15. Jaenike J, Unckless $R$, Cockburn SN, Boelio LM, Perlman SJ: Adaptation via symbiosis: recent spread of a Drosophila defensive symbiont. Science 2010, 329:212-215

16. Xie JL, Vilchez I, Mateos M: Spiroplasma bacteria enhance survival of Drosophila hydei attacked by the parasitic wasp Leptopilina heterotoma. Plos One 2010, 5:e12149.

17. Hedges LM, Brownlie JC, O'Neill SL, Johnson KN: Wolbachia and virus protection in insects. Science 2008, 322:702-702.

18. Teixeira L, Ferreira A, Ashburner M: The bacterial symbiont Wolbachia induces resistance to RNA viral infections in Drosophila melanogaster. Plos Biology 2008, 6:2753-2763.

19. Osborne SE, Leong YS, O'Neill SL, Johnson KN: Variation in antiviral protection mediated by different Wolbachia strains in Drosophila simulans. Plos Pathogens 2009, 5:11.

20. Moreira LA, Iturbe-Ormaetxe I, Jeffery JA, Lu G, Pyke AT, Hedges LM, Rocha BC, Hall-Mendelin S, Day A, Riegler M, et al: A Wolbachia symbiont 
in Aedes aegypti limits infection with dengue, Chikungunya, and Plasmodium. Cell 2009, 139:1268-1278.

21. Bian $G, X u Y, L u P, X i e$ Y, Xi Z: The endosymbiotic bacterium Wolbachia induces resistance to dengue virus in Aedes aegypti. PLOS Pathog 2010, 6:e1000833.

22. Frentiu FD, Robinson J, Young PR, McGraw EA, O'Neill SL: Wolbachiamediated resistance to dengue virus infection and death at the cellular level. Plos One 2010, 5:e13398.

23. Glaser RL, Meola MA: The native Wolbachia endosymbionts of Drosophila melanogaster and culex quinquefasciatus increase host resistance to west Nile Virus Infection. Plos One 2010, 5.

24. Himler AG, Adachi-Hagimori T, Bergen JE, Kozuch A, Kelly SE, Tabashnik BE, Chiel E, Duckworth VE, Dennehy TJ, Zchori-Fein E, Hunter MS: Rapid spread of a bacterial symbiont in an invasive whitefly is driven by fitness benefits and female bias. Science 2011, 332:254-256.

25. Caspari E, Watson GS: On the evolutionary importance of cytoplasmic sterility in mosquitos. Evolution 1959, 13:568-570.

26. Turelli M: Evolution of incompatibility-inducing microbes and their hosts. Evolution 1994, 48:1500-1513.

27. Hoffmann AA, Clancy DJ, Merton E: Cytoplasmic incompatibility in Australian populations of Drosophila melanogaster. Genetics 1994, 136:993-999.

28. Solignac M, Vautrin D, Rousset F: Widespread occurrence of the proteobacteria Wolbachia and partial cytoplasmic incompatibility in Drosophila-Melanogaster. Comptes Rendus De L Academie Des Sciences Serie lii-Sciences De La Vie-Life Sciences 1994, 317:461-470.

29. Hoffmann AA, Turelli M: Cytoplasmic incompaibility in insects. In Influential passengers: inherited microorganisms and arthropod reproduction. Oxford University Press;O'Neil S, Hoffmann AA, Werren JH 1997:42-80.

30. Fenton A, Johnson KN, Brownlie JC, Hurst GD: Solving the Wolbachia paradox: modeling the tripartite interaction between host, Wolbachia, and a natural enemy. Am Nat 2011, 178:333-342.

31. Jiggins FM, Hurst GD, Jiggins $C D$, $v$ d Schulenburg JH, Majerus ME: The butterfly Danaus chrysippus is infected by a male-killing Spiroplasma bacterium. Parasitology 2000, 120(Pt 5):439-446.

32. Duron O, Bouchon D, Boutin S, Bellamy L, Zhou LQ, Engelstadter J, Hurst GD: The diversity of reproductive parasites among arthropods: Wolbachia do not walk alone. BMC Biology 2008, 6.

33. Hurst GDD, Johnson AP, von der Schulenburg JHG, Fuyama Y: Male-killing Wolbachia in Drosophila: a temperature-sensitive trait with a threshold bacterial density. Genetics 2000, 156:699-709.

34. Index of viruses - Dicistroviridae. In ICTVdB - The Universal Virus Database, version 4. Columbia University, New York, USA;Büchen-Osmond, C [http:// www.ncbi.nlm.nih.gov/ICTVdb/lctv/fs_index.htm].

35. Brun G, Plus N: The viruses of Drosophila. In The genetics and biology of Drosophila. New York: Academic Press;Ashburner M, Wright TRF 1980:625-702

36. Johnson KN, Christian PD: Molecular characterization of Drosophila C virus isolates. J Invertebr Pathol 1999, 73:248-254.

37. Kapun M, Nolte V, Flatt $T$, Schlotterer $C$ : Host range and specificity of the Drosophila C Virus. Plos One 2010, 5:e12421.

38. Jousset FX: Host range of Drosophila-Melanogaster C Virus among Diptera and Lepidoptera. Annales De Microbiologie 1976, A127:529-\&.

39. Index of viruses - Nodaviridae. In ICTVdB - The Universal Virus Database, version 4. Columbia University, New York USA;Büchen-Osmond, C [http:// www.ncbi.nlm.nih.gov/lCTVdb/lctv/fs index.htm]

40. Scotti PD, Dearing S, Mossop DW: Flock house virus - a Nodavirus isolated from Costelytra-Zealandica (White) (Coleoptera, Scarabaeidae). Archives of Virology 1983, 75:181-189.

41. Dasgupta R, Cheng LL, Bartholomay LC, Christensen BM: Flock house virus replicates and expresses green fluorescent protein in mosquitoes. Journal of General Virology 2003, 84:1789-1797.

42. Dasgupta R, Free HM, Zietlow SL, Paskewitz SM, Aksoy S, Shi L, Fuchs J, Hu C, Christensen BM: Replication of flock house virus in three genera of medically important insects. J Med Entomol 2007, 44:102-110.

43. Price $B D$, Rueckert RR, Ahlquist $P$ : Complete replication of an animal virus and maintenance of expression vectors derived from it in Saccharomyces cerevisiae. Proc Natl Acad Sci U S A 1996, 93:9465-9470.

44. Selling BH, Allison RF, Kaesberg P: Genomic RNA of an insect virus directs synthesis of infectious virions in plants. Proc Natl Acad Sci U S A 1990 87:434-438.
45. Longdon B, Wilfert L, Obbard DJ, Jiggins FM: Rhabdoviruses in two species of Drosophila: vertical transmission and a recent sweep. Genetics 2011, 188:141-150.

46. Galiana-Arnoux D, Dostert C, Schneemann A, Hoffmann JA, Imler JL: Essential function in vivo for Dicer-2 in host defense against RNA viruses in drosophila. Nat Immunol 2006, 7:590-597.

47. Reed $\amalg$, Muench $\mathrm{H}$ : A simple method of estimating fifty per cent endpoints. The American Journal of Hygiene 1938, 27:493-497.

48. Klohn PC, Stoltze L, Flechsig E, Enari M, Weissmann C: A quantitative, highly sensitive cell-based infectivity assay for mouse scrapie prions. Proc Natl Acad Sci U S A 2003, 100:11666-11671.

49. Sullivan W, Ashburner M, Hawley S: Drosophila Protocols. Cold Spring Harbor Laboratory Press; 12000.

50. Baldo L, Dunning Hotopp JC, Jolley KA, Bordenstein SR, Biber SA, Choudhury RR, Hayashi C, Maiden MC, Tettelin H, Werren JH: Multilocus sequence typing system for the endosymbiont Wolbachia pipientis. Appl Environ Microbiol 2006, 72:7098-7110.

51. Sheeley SL, MCAllister BF: Mobile male-killer: similar Wolbachia strains kill males of divergent Drosophila hosts. Heredity 2009, 102:286-292.

52. Jiggins FM, von der Schulenburg JHG, Hurst GDD, Majerus MEN Recombination confounds interpretations of Wolbachia evolution. Proceedings of the Royal Society B-Biological Sciences 2001, 268:1423-1427.

53. Werren JH, Bartos JD: Recombination in Wolbachia. Current Biology 2001, 11:431-435.

54. Masui S, Kamoda S, Sasaki T, Ishikawa H: Distribution and evolution of bacteriophage WO in Wolbachia, the endosymbiont causing sexual alterations in arthropods. J Mol Evol 2000, 51:491-497.

55. Oliver KM, Degnan PH, Hunter MS, Moran NA: Bacteriophages encode factors required for protection in a symbiotic mutualism. Science 2009, 325:992-994.

doi:10.1186/1471-2180-12-S1-S8

Cite this article as: Longdon et al:: Male-killing Wolbachia do not protect Drosophila bifasciata against viral infection. BMC Microbiology 2012 12(Suppl 1):S8.

\section{Submit your next manuscript to BioMed Central and take full advantage of:}

- Convenient online submission

- Thorough peer review

- No space constraints or color figure charges

- Immediate publication on acceptance

- Inclusion in PubMed, CAS, Scopus and Google Scholar

- Research which is freely available for redistribution 